\title{
Aspergillus in liquid-based cervicovaginal cytology in a postmenopausal patient: A case report
}

\author{
RAQUEL GARZA-GUAJARDO ${ }^{1}$, LUIS CARLOS CANALES-MARTÍNEZ ${ }^{1}$, \\ IRÁM PABLO RODRÍGUEZ-SÁNCHEZ², MARÍA MARISELA SÁNCHEZ-CHAPARRO ${ }^{3}$, \\ GABRIELA SOFÍA GÓMEZ-MACÍAS ${ }^{1}$, NATALIA VILCHES-CISNEROS ${ }^{1}$ and ORALIA BARBOZA-QUINTANA ${ }^{1}$ \\ ${ }^{1}$ Department of Pathological Anatomy and Cytopathology, Universidad Autonoma de Nuevo Leon, \\ Hospital Universitario 'Dr José Eleuterio González'; ${ }^{2}$ Department of Genetics, Universidad Autonoma \\ de Nuevo Leon, College of Medicine, Monterrey, Nuevo León 64460; ${ }^{3}$ Laboratory of Immunology \\ and Virology, Universidad Autonoma de Nuevo Leon, College of Biological Sciences, \\ San Nicolás de los Garza, Nuevo León 66450, Mexico
}

Received January 13, 2017; Accepted February 16, 2017

DOI: $10.3892 / b r .2017 .974$

\begin{abstract}
Aspergillus is an opportunistic fungus present in humid environments, whose natural environment is in soil, hay and compost. It is a frequent contaminant in the clinical laboratory. Because of this, the fungus is often inhaled, affecting those with an underlying pulmonary disease or immune deficiency. Fungal genitourinary tract infections are relatively common. A rare Aspergillus spp cervical infection diagnosed via liquid-based cytology is presented in the current study. The 57-year-old woman attended her annual check-up without any relevant medical history. The result of a gynecological examination by Papanicolaou smear was normal and routine liquid-based cytology was performed. The specimen exhibited fungal organisms characterized by septate hyphae branching at acute angles, most consistent with the Aspergillus species. Subsequent cytology demonstrated the same results. Antifungal treatment was initiated and a second post-treatment smear only exhibited atrophy. The cytomorphological features of Aspergillus spp. are discussed in the current study and a brief review of the few reported cases of a primary cervical infection in the literature is provided. In addition, the liquid-based cytology was established as a tool to diagnose the rare Aspergillus infection.
\end{abstract}

Correspondence to: Dr Oralia Barboza-Quintana, Department of Pathological Anatomy and Cytopathology, Universidad Autonoma de Nuevo Leon, Hospital Universitario 'Dr José Eleuterio González', Francisco I. Madero y Gonzalitos S/N, Mitras Centro, Monterrey, Nuevo León 64460, Mexico

E-mail: oralibarboza@gmail.com

Key words: Aspergillus, liquid-based cervicovaginal, smear, cytology, diagnosis

\section{Introduction}

The most relevant of the Aspergillus species are fumigatus, flavus and niger. These grow in the natural environment, and in tissues and cultures in the form of hyphae that produce conidia upon exposure to air. Conidia consist of a conidiophore with a terminal vesicle in which one or two layers of phialides are developed. The prevalence of genitourinary tract infections has been reported to be as high as $52 \%$ in the female population, with the most common cause being Candida albicans in 28\% (1). With the exception of Candida, other fungi are rarely observed in Papanicolaou (Pap) smears or biopsies, with isolated reports of opportunistic infections, such as Blastomyces dermatitidis, Coccidioides immitis, Cryptococcus neoformans and mucor being published $(2,3)$. To the best of our knowledge, this is one of few Aspergillus infection cases reported regarding infection by Aspergillus as diagnosed via liquid-based gynecologic cytology, including a clinical and cytological follow-up.

\section{Case report}

The patient was a 57-year-old Mexican woman who had been through the menopause five years previously and she had no relevant medical history. During an annual medical checkup in July 2015, the patient went to the gynecology service at the Hospital Universitario 'Dr José Eleuterio González' (Monterrey, Mexico). She presented without any signs, symptoms or concerns. Furthermore, previous checkups revealed no anomalies. However, the physical examination revealed an atrophic state in the vagina and cervix.

Previous patient authorization, a routine liquid-based cervical cytology was performed and demonstrated atrophy with a predominance of parabasal cells and scarce endocervical cells. Of note, multiple fungal structures with uniform and homogeneous forms, and a parallel contour were identified; septate hyphae, and acute-angle branching $\left(45^{\circ}\right)$ consistent with Aspergillus spp. were observed too. Terminal vesicles 

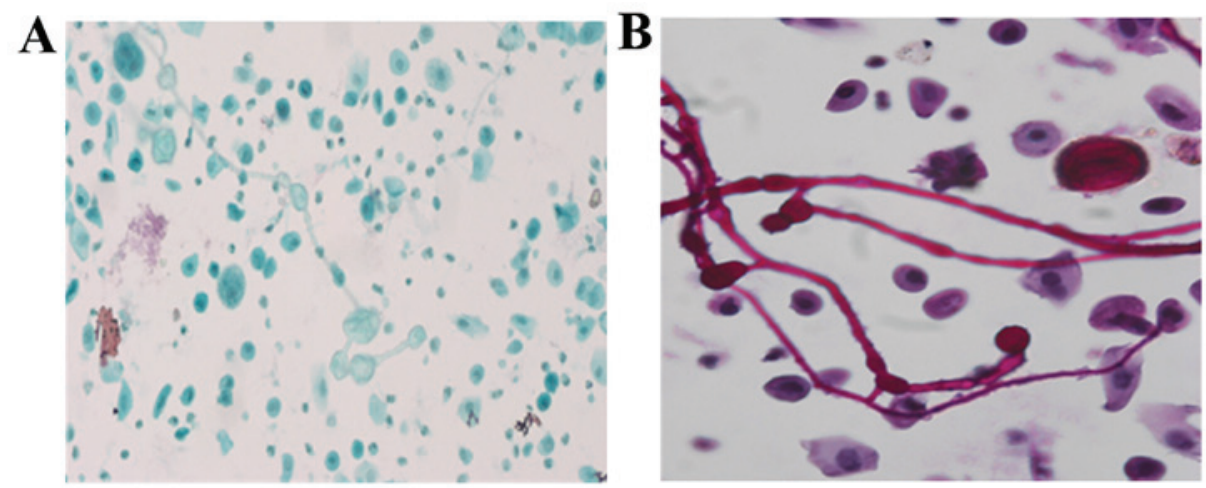

Figure 1. Liquid-based cytology. (A) Dichotomous branching of septate hyphae in Aspergillus (Papanicolaou staining using the PAS technique; magnification, $\mathrm{x} 4$ ). (B) PAS staining highlights these characteristics (magnification, x40). PAS, Periodic Acid-Schiff.
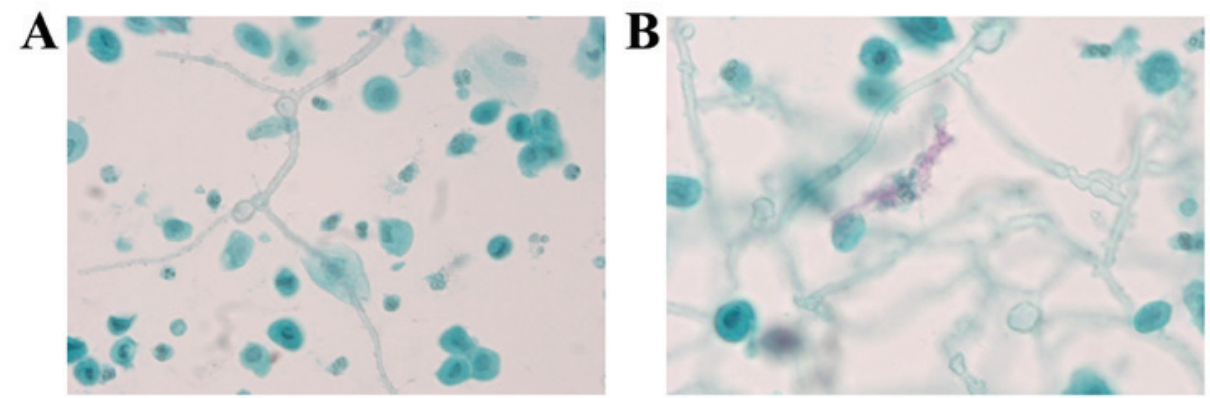

Figure 2. Verification of the presence of Aspergillus. The sample was further analyzed by PAS staining. These correspond to the Aspergillus structures previously observed in Fig. 1. (A) Magnification, x4. (B) Magnification, x40.
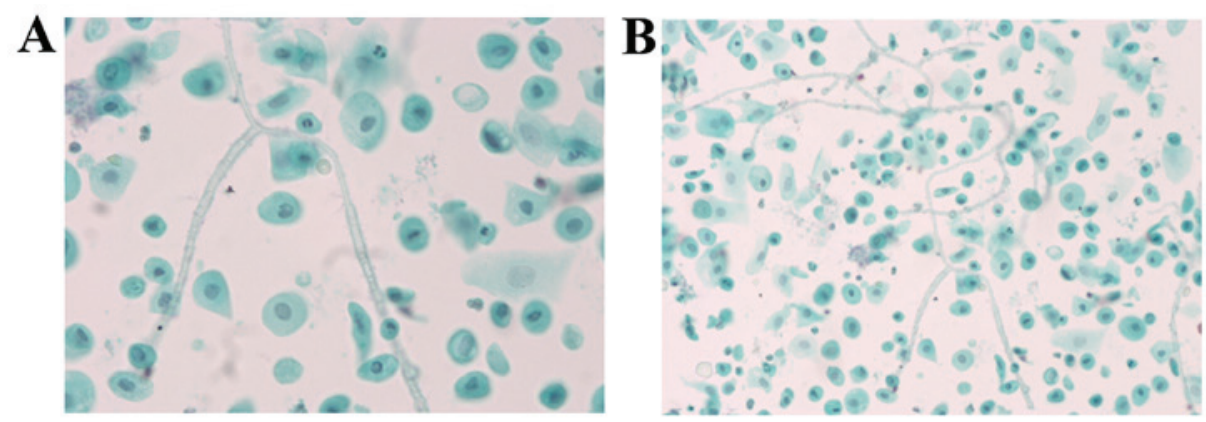

Figure 3. Sample obtained 10 days after the first stains. PAS technique of the progressive acute angle dichotomous branching of Aspergillus. The possibility of previous cross-contamination was discarded. (A) Magnification, x40. (B) Magnification, x4.

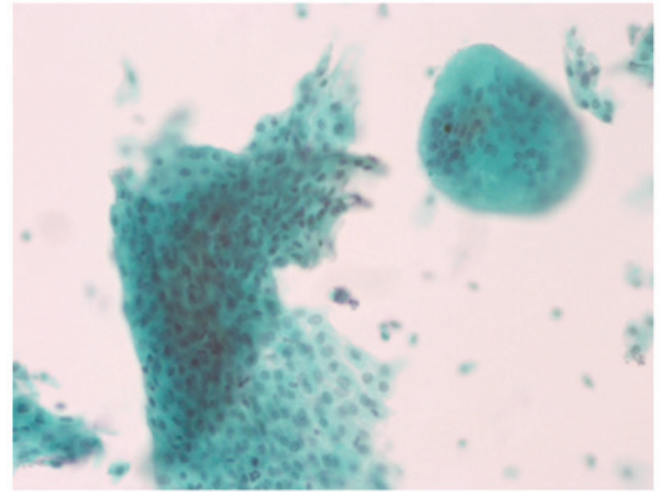

Figure 4. Post-treatment smear test (third sample). Atrophic smear, exhibiting an increased number of basal and parabasal cells in sheets with a clean background. PAS technique; magnification, $\mathrm{x} 4$. were identified, but there were no phialides. The Aspergillus' morphology was accompanied by features of severe chronic and acute inflammation, foreign body giant cells and numerous benign squamous cells. The squamous cells and fungal structures were situated in the same plane.

As laboratory contamination is a diagnostic challenge, and even though fungal structures were observed in the same plane as cells, a novel aliquot was created from the same sample (Fig. 1). The second slide demonstrated the same findings and Periodic acid-Schiff (PAS) staining was used to highlight the microorganisms (Fig. 2). As the patient was asymptomatic it was decided to obtain a novel sample 10 days later to rule out cross-contamination. This second sample again presented numerous hyphae and vesicles consistent with Aspergillus (Fig. 3). Post-treatment cytology, 
also liquid-based, exhibited large numbers of parabasal cells, a cleaner background, multinucleated giant cells and an absence of fungi (Fig. 4). Treatment was initiated with vaginal itraconazole for seven days, although the patient was asymptomatic. The only risk factor that was established was occasional cleansing of the genital area after cleansing of the nostrils, using the same handkerchief.

The fruiting bodies of Aspergillus provide information about this fungus and its species. Identification of the length and size of the conidiophore, as well as the shape of the vesicles of the conidia, are important. In histological sections and smears, thick and uniform septate hyphae (3-6 $\mu \mathrm{m})$ are observed. In addition, a dichotomy division at an angle of $45^{\circ}$ is characteristic (4). These findings correlate with the fungus that was observed in multiple smears in the present study. The fungus is easily observed using a Pap smear; however, stains for fungi, such as PAS and Grocott, highlight the morphology. Conidia are rarely observed in tissue samples in human infections, as these forms when the fungus is exposed to air.

Conidia are observed in conditions, such as fungus ball/ aspergillomas. The observation of Aspergillus in a Pap smear is rare. It can represent a symptomatic infection in immunosuppressed patients or patients undergoing prolonged antibiotic treatment or in patients with a contamination. Previous studies have reported that equipment used for the Pap test (vaginal mirrors, foil and spray attachments) may be contaminated (5-8). In the current case, the quantity of pseudo-hyphae and the presence of a significant chronic inflammatory infiltrate, in addition to its position in the same plane as the epithelial cells, indicated that this was a genuine infection.

\section{Discussion}

There are previous reports on the presence of Aspergillus in smear tests. Certain reports describe cervical smears where the authors conclude that the results could be due to contamination, as the other five slides exhibited the same hyphae $(5,6)$. Additional case reports exhibited a smear test result with a description of a fruiting body in a patient $(6,7)$. Deb and Srivastava (3) and Gupta et al (5) reported concomitant Aspergillus in patients with high-grade intraepithelial lesions, which could be explained by their immunocompromised state $(3,5,9)$. To the best of our knowledge, Hoda et al (10) are the only other authors to report on liquid-based Aspergillus, identifying only one fruiting body; however, they do not report any inflammatory infiltrate or hyphae that could clarify whether the finding was due to contamination (10).
Identification of a fungus that tends to cause systemic infection alerts the attending physician to look for and identify risk factors, specifically factors that lead to immunocompromise. In the current case, all the necessary precautions were taken to identify whether this was truly an infection rather than a contamination. Initially, all slides that were processed immediately were evaluated to observe any pseudo-hyphae, in addition to repeating the liquid-based cytology to rule out equipment contamination in the cytology department. Finally, a second cytology specimen was performed with completely different equipment from that which was used during the first sample. Once the diagnosis of Aspergillus was confirmed, adequate treatment was administered. All appropriate measures were taken to confirm that the observation of cervicovaginal Aspergillus infection from liquid-based cytology and a Pap smear was not due to contamination.

\section{Acknowledgements}

The authors would like to thank Dr Sergio Lozano-Rodríguez for the critical reading of the manuscript.

\section{References}

1. Sullam SA, Mahfouz AA, Dabbous NI, el-Barrawy M and el-Said MM: Reproductive tract infections among married women in Upper Egypt. East Mediterr Health J 7: 139-146, 2001

2. Sheyn I, Mira JL and Thompson MB: Paracoccidioides brasiliensis in a postpartum Pap smear. A case report. Acta Cytol 45: 79-81, 2001.

3. Deb P and Srivastava A: Aspergillus in a cervico-vaginal smear of an adult postmenopausal female: An unusual case. J Cytol 26: 123-124, 2009.

4. Donta B, Naik DD, Mali BN, Bandiwadekar A, Ramnath K and Rao M: 'Fruiting body' Penicillium species: Papanicolaou stained conventional cervical smear findings. Diagn Cytopathol 38: 34-35, 2010.

5. Gupta P, Goyal S and Kaushal M: Concomitant aspergillus species infection and squamous cell carcinoma diagnosed on Pap smear. Turk Patoloji Derg 32: 54-56, 2016.

6. Brimo F, Ouad L, Brodeur J, Charbonneau M and Auger M: Unusual microbial organisms seen in two cervical smears. Diagn Cytopathol 37: 836-838, 2009.

7. Martínez-Girón R and Fernández-García C: Aspergillus/Penicillium sp. spores as a contaminant on conventional Pap smear. Diagn Cytopathol 37: 899-900, 2009.

8. Policarpio-Nicolas ML, Covell J, Moore K and Stelow EB: 'Paintbrush' appearance of Penicillium species in Thin-Prep cervico-vaginal (Pap) test. Diagn Cytopathol 36: 721-722, 2008.

9. Chandra S, Gaur D, Harsh M, Chaturvedi J and Kishore S: An unusual presentation of Aspergillus species in a routine cervicovaginal pap smear: A case report. Acta Cytol 53: 229-231, 2009.

10. Hoda RS, Colello C, Roddy M and Houser PM: 'Fruiting body' of Aspergillus species in a routine cervico-vaginal smear (Pap test). Diagn Cytopathol 33: 244-245, 2005. 